\title{
Concussion among soccer players in the 2017 Brazilian championship - the gap between protocol and medical practice
}

\author{
Cármine Porcelli Salvarani*, (1D), Lucas Ribeiro de Medeiros' ${ }^{1}$, Fernando Henrique Sapatero', \\ Diego Ciotta de Castro ${ }^{1}$, Vinícius Simon Tomazini ${ }^{1}$, Leonardo Henrique Micheletti \\ Sotocorno1, Paulo Sérgio Teixeira da Costa ${ }^{1}$, Bruno Bueno Pimenta1, Diego Almeida de \\ Oliveira1 ${ }^{1}$ Eduardo Almeida Dias ${ }^{1}$ \& Eduardo Vinícius Colman da Silva ${ }^{1}$ \\ ${ }^{1}$ State University of Maringá, Medicine Department - Division of Neurosurgery, Avenida Mandacaru 1590, Bloco S 05, Sala 10, \\ Hospital Universitário, Maringá, PARANÁ, CEP 87083-240, Brazil \\ *Author for correspondence: Tel.: +55 44991186 718; cpsalvarani@gmail.com
}

Background: The present study aims to report traumatic brain injury (TBI) among soccer players in the 2017 Brazilian Soccer Championship and discuss the protocols for concussion evaluation. Materials \& methods: This is an observational study utilizing video analysis of 380 matches. TBI was considered as any event in which one or more soccer player(s) had a head trauma. For potential concussion diagnosis, we analyzed players with one of the following signs: slowness to get up, disorientation, motor incoordination, loss of consciousness, head clutching and impact seizure. Results: There were 374 TBIs in total. The average time for medical assessment was 1'35". 13 players had concussion with an average time of 3'19" for medical evaluation. Four players were replaced after having a concussion. Conclusion: There is a gap between concussion protocols and medical practices in Brazilian elite soccer. Further discussion about soccer replacement rules are imperative.

First draft submitted: 13 September 2020; Accepted for publication: 5 October 2020; Published online: 28 October 2020

Keywords: soccer • sports-related concussion • traumatic brain injury • video analysis

Sports-related concussion (SRC) is a worldwide public health concern. Concussion is a subset of mild traumatic brain injury (TBI) and it is defined as traumatically induced transient disturbance of the brain function that involves a complex pathophysiological process. Based on acute injury characteristics, concussion is placed at the less severe end of the brain injury spectrum [1].

In the world of sports, the highest incidence of SRC occurs in American Football, hockey, rugby, soccer and basketball. In soccer, head injury can be the result of a collision between two heads (or other body parts), or between a head and the ground, goal post, other unknown object or an unexpected ball hit. Due to the global popularity of soccer, the overall contribution of this sport to the total SRC amount is likely to be significantly more than other sports [2].

According to recent publications, the neurological understanding of SRC consequences and its effect on the lives of athletes, especially regarding soccer, is still not complete. As a result, the development of effective tools for proper management of these injuries has been reported by current research. The sideline assessment of SRC in soccer is challenging due to the variability of its clinical presentation, the specific environment, the reliance on athlete-reported symptoms and the varying specificity and sensitivity values of sideline assessment tools. The correct assessment of suspected concussion immediately after injury is an important practice for early diagnosis, for appropriate management of athletes with TBI and for determining whether it is safe for a player to return to the match $[3,4]$.

There are protocols provided by the consecutive 3rd, 4th and 5th International Conferences on Concussion in Sports that clinicians are recommended to follow when an athlete shows any sign of concussion [5-7]. The latest Sport Concussion Assessment Tool (SCAT), developed by Consensus Statement on Concussion in Sports, was

Future $\because$ Medicine 


\begin{tabular}{|l|l|}
\hline Round & Match teams \\
\hline Match date and time & Responsible \\
\hline Number of TBls & Minute/half \\
\hline Etiology & Cut blunt injury () yes () no \\
\hline Head $\times$ head (double TBI) & \\
\hline Head $\times$ hand & Conclusion () yes () no \\
\hline Head $\times$ foot & Duration of medical assessment (if it occurs) \\
\hline Head $\times$ elbow & \\
\hline Head $\times$ knee & Soccer replacement () yes () no \\
\hline Head $\times$ goal post & If yes, reason: () Concussion \\
\hline Head $\times$ ground & \multicolumn{1}{c|}{ () Cut blunt injury } \\
\hline Head $\times$ leg & () Both \\
\hline Head $\times$ hip & \\
\hline Head $\times$ ball & Observations \\
\hline Others & \\
\hline
\end{tabular}

Figure 1. Protocol for collection of data for traumatic brain injury.

adopted by Fédération Internationale de Football Association (FIFA; Zürich, Switzerland) [8,9]. However, previous reports using video analysis from the 2014 Men's FIFA World Cup and the 2016 Men's Union of European Football Association (UEFA) found that many potential concussion events were not correctly evaluated, showing a lack of congruence between these protocols and practices on field [10,11].

This study is the first specific report with video analysis of TBI among soccer players in the Brazilian Series A Soccer Championship, regarding the complete epidemiology, undertaken with an aim to verify the characteristics of TBI in Brazilian Elite soccer and to discuss the medical protocols for concussion in soccer matches.

\section{Materials \& methods}

This is an observational study concerning video analysis of all 380 matches (38 rounds, 10 matches each round) of the 2017, Series A, Brazilian Soccer Championship, based on recent publications related to SRC with video analysis [10-14]. The videos of players with suspected TBI were first analyzed at the same time the matches took place by a team of ten trained medical students of the State University of Maringá (Paraná, Brazil), under the guidance of a neurosurgery professor. After first analysis, all suspected TBIs were reviewed by the research coordinator.

\section{Guideline \& coding of events}

A guideline was built to record data about TBI characteristics (Figure 1). In order to fulfill the guideline, TBI was considered as any event in which one or more soccer players had a head trauma (head $\times$ head, head $\times$ unexpected ball hit or head $\times$ elbow, for instances). On multiple occasions, a Brazilian soccer player claimed to have a TBI, but they were pretending to gain an advantage in the match. To minimize this situation, all videos with suspected TBI were inserted in a smartphone application platform (WhatsApp ${ }^{\circledR}$, Facebook, CA, USA), shared among all students and checked by the research coordinator. In addition to the videos, the television match commentary was taken to add some information about TBI. A 'head-to-head' TBI was considered a double TBI, meaning two TBIs occurring at the same moment.

While we acknowledge that concussions can also occur from contact to other parts of the body, besides the head and neck area, this study limited the assessment of concussions only after direct trauma with the head area. A potential concussion diagnosis was defined as any event of TBI (through direct contact among athletes, unexpected ball hit or object in the environment) that left the soccer player unable to immediately resume play following impact. Further, for concussion diagnosis, we analyzed players with one of these visual signs: slowness to get up, disorientation, motor incoordination, loss of consciousness, head clutching and impact seizure. When a soccer player was replaced due to TBI, the after-match medical information was taken from media information and/or from the official soccer team medical statement. 
Table 1. Characteristics of traumatic brain injury concerning overall events, relation with matches, etiology, cut blunt injury, concussion and players' replacements (total number of matches: 380 , total number of traumatic brain injury: 374).

\begin{tabular}{|c|c|c|c|}
\hline TBI per match & 0.98 & Matches with TBI & 195 \\
\hline TBI per round & 9.98 & Matches without TBI & 185 \\
\hline Etiology & & Cut blunt wound & 30 \\
\hline - head $\times$ head (double TBI) & $91(182)$ & - head $\times$ head & 19 \\
\hline - head $\times$ elbow & 86 & - head $\times$ elbow & 6 \\
\hline - head $\times$ foot & 29 & - head $\times$ foot & 4 \\
\hline - head $\times$ unexpected ball hit & 17 & - head $\times$ knee & 1 \\
\hline - head $\times$ knee & 12 & & \\
\hline - head $\times$ leg & 11 & Player replacement after TBI & 12 \\
\hline - head $\times$ shoulder & 10 & & \\
\hline - head $\times$ hip & 9 & Concussion ( $3.5 \%$ of all TBI) & 13 \\
\hline - head $\times$ hand & 7 & & \\
\hline - head $\times$ ground & 4 & Player replacement after concussion & 4 \\
\hline - head $\times$ others & 7 & & 1 \\
\hline
\end{tabular}

\section{Medical assessment}

The length of sideline medical assessment was systematically recorded in all cases of TBI that required a medical attendance. The start of medical assessment was considered to be when medical staff reached the player, whether on the field or on the sidelines. The end of the medical assessment was established by the medical decision of the player returning to play or being replaced.

\section{Patient \& public involvement}

Patients and/or public were not involved in this research.

\section{Results}

Across the 380 matches a total of 374 TBIs occurred, averaging almost one TBI per match (0.98). There were 30 cut blunt injuries among TBIs and three players were replaced to have wounds sutured due to the extension of their injuries. The other 27 players were treated by compressive dressing with swimming caps and were not replaced. The overall findings, with etiology, cut blunt injuries, concussion and players' replacements are shown on Table 1.

Thirteen players had concussion (3.5\% of all TBIs) and only four of them were replaced. Of these players who had concussion, only two were assisted by an Ambulance in the field. These two players had impact seizure and, in both situations, were removed to a hospital (Figure 2). The post match computed tomography and MRI of both players were normal.

The maximum number of TBIs in one match was seven, but no players were replaced in that match. Three of those TBIs occurred in just one play that involved three players (a triple head TBI).

There were 82 events of TBI where medical assessment was not required. In general, the mean time for medical assessment among the remaining 292 TBIs was 1'35" (from 48" to 7'40"). Considering the 13 players who had concussion and the 12 players who were replaced (four due to concussion, four due to head cut blunt injury, two due to dizziness, two due headache), the mean time for medical evaluation was $3^{\prime} 26^{\prime \prime}$ and $3^{\prime} 19^{\prime \prime}$, respectively (see Tables $2 \& 3$ ). If the two reported severe TBIs, for whom an ambulance was required on field, were excluded from the average, the mean time for medical evaluation goes down to $2^{\prime} 43$ " for players with concussion and $2^{\prime} 54$ " for replaced players after TBI. In both situations, the length of medical evaluation was very similar despite the occurrence of concussion. Comparing the overall mean time for medical assessment and players with concussion, there was an increase of $1^{\prime} 51$ ". 3.

The main characteristics of players with concussion and replaced players after TBI are described on Tables $2 \&$ 


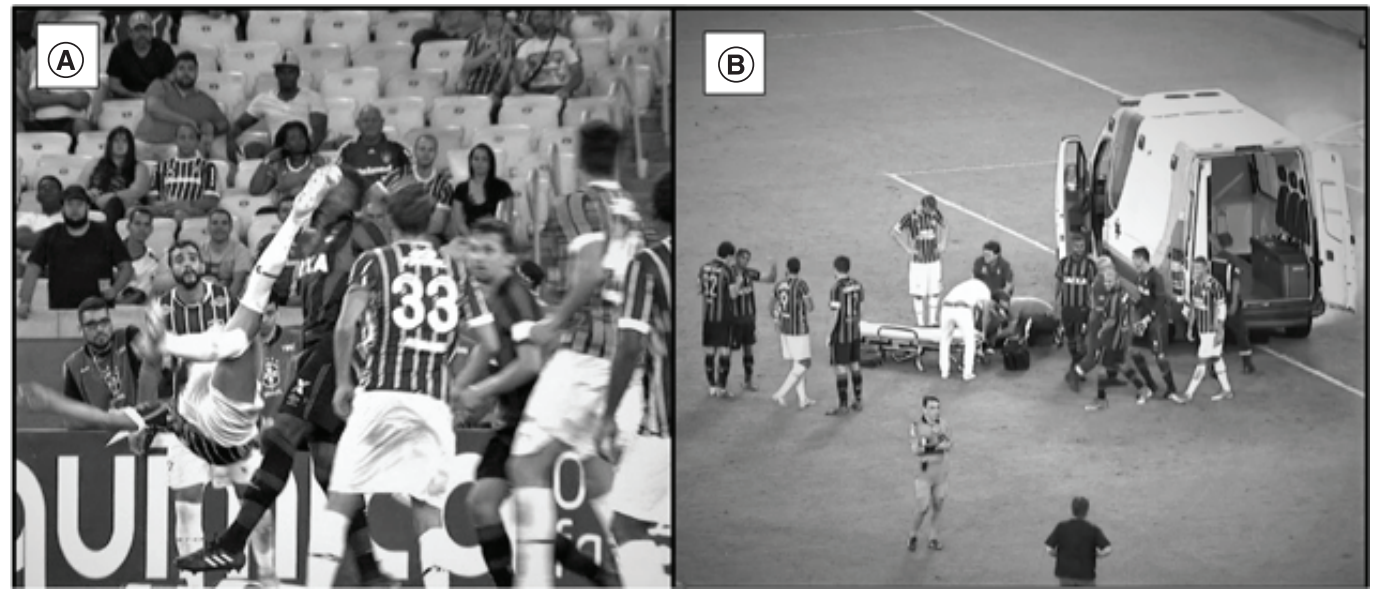

Figure 2. Player with concussion after head $x$ foot trauma. (A) Player's photo showing the moment of traumatic brain injury with later impact seizure. (B) Ambulance on the field during medical assessment to remove the player to a hospital.

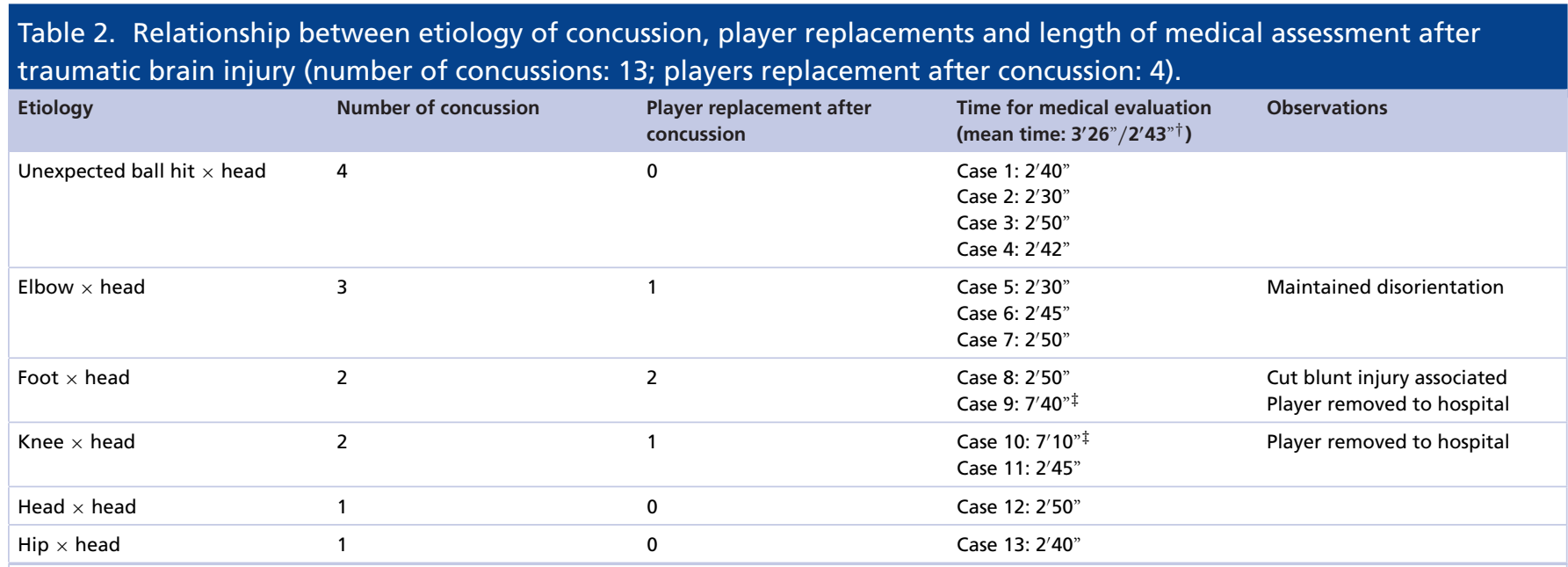

$\dagger$ Mean time for medical evaluation without cases 9 and 10 .

¥In both situations, players were removed from field by ambulance to hospital.

\begin{tabular}{|c|c|c|c|}
\hline Etiology & $\mathrm{n}$ & Cause/observation & $\begin{array}{l}\text { Time for medical evaluation } \\
\left.\text { (mean time: } 3^{\prime} 19^{\prime \prime} / 2^{\prime} 54^{\prime \prime}\right)\end{array}$ \\
\hline Foot $\times$ head & 2 & $\begin{array}{l}\text { Case 2: impact seizure/player remove to hospital } \\
\text { Case 3: concussion/cut blunt injury associated }\end{array}$ & $\begin{array}{l}7^{\prime} 40^{\prime \prime} \\
2^{\prime} 50 "\end{array}$ \\
\hline Elbow $\times$ head & 4 & $\begin{array}{l}\text { Case 8: concussion/maintained disorientation } \\
\text { Case 9: cut blunt injury } \\
\text { Case 10: cut blunt injury } \\
\text { Case 11: headache }\end{array}$ & $\begin{array}{l}2 ' 30 " \\
1^{\prime} 45^{\prime \prime} \\
1^{\prime} 40^{\prime \prime} \\
2^{\prime} 20^{\prime \prime}\end{array}$ \\
\hline Shoulder $\times$ head & 1 & Case 12: headache & $2^{\prime} 40^{\prime \prime}$ \\
\hline
\end{tabular}




\section{Discussion}

In the history of soccer, there have been reports of some unforgettable TBI occurrences: Leonardo and Tab Ramos, in a match between Brazil and USA at the 1994 FIFA World Cup; Petr Cech, goalkeeper of Chelsea, during a match against Reading in the 2006 Premier League Championship; and Fernando Torres at the La Liga Championship in 2017. In Brazil, a soccer player called Vagner Bacharel died 6 days after a TBI during a match in 1990. The misdiagnosed acute epidural hematoma was the etiology of his death. Year after year, TBI among soccer players has been growing and the concern with their assessment has been reported [15-18].

Video analysis does not provide full information about TBI and medical assessment; however, despite its limitations, it may be a useful adjunct to the sideline assessment of a possible concussion [12]. In the National Football League, a very recent study showed that visual signs for potential concussion detected by video analysis cannot be made on-field alone. The video analysis is a part of the comprehensive sideline/acute evaluation of concussion and the diagnosis remains a multimodal clinical decision [19].

Recent studies utilized video analysis at the 2014 Men's FIFA World Cup and the 2016 UEFA European Championship [10,11]. According to these publications, this first study among soccer players in Brazil, supported by video analysis, showed how the concussion was evaluated on sideline. In addition, it was studied the overall epidemiologic data of TBI, the length of medical assessment of players who had concussion and/or were replaced after TBI.

In terms of soccer biomechanics, head-to-head impacts and unexpected ball hits with great speed each result in the greatest form of head acceleration and, consequently, come with higher risks of concussion $[2,20]$. This is important because, in our findings, the most frequent TBI event was head-to-head (91 events resulting in 182 TBIs); however, there were four concussions after an unexpected ball hit (out of 17 events) and only one case of concussion after head-to-head impact.

Updated protocols for sideline assessment of an athlete with a concussion were published following each of the International Conferences on Concussion in Sport in 2008, 2012 and 2016 [5-7]. The Concussion in Sports Group recommendations assert that when an athlete shows any features of a concussion, the athlete should be evaluated by a physician or another licensed healthcare provider onsite, assessed using SCAT or other sideline concussion assessment tools and prevented from return to play in the event of a positive diagnosis. The SCAT-5 is the assessment tool supported and endorsed by FIFA [9].

In the Men's 2014 FIFA World Cup and 2016 UEFA European Championship, 63 and 72.5\% of athletes who experienced a potential concussion event were not medically assessed, respectively. All players with a potential concussion event that were medically assessed resulted in no replacement after TBI [10,11].

In this study, only four players out of 13 with a concussion during the Championship were replaced on the pitch. This included two players who were assisted by an ambulance on the field and removed directly to the hospital due to an impact seizure and one player who had an extensive cut blunt injury associated with the concussion. Just one player was replaced after the concussion without any associated injuries. Besides, on two different occasions, video images showed that the decision to return to the match was motivated and pressured more by the athlete's desire than the medical staff itself.

Soccer officials should be trained to recognize signs and symptoms of concussion in order to know when to ask for a medical assessment [21]. After that, physicians must provide care along the continuum of the concussion, from the acute injury to return-to-play decisions [2]. According to SCAT-5, which has been adopted by FIFA [9], the protocols cannot be performed in less than $10 \mathrm{~min}$. However, during the 2014 FIFA World Cup Championship, the mean time for player assessment after suspected concussion was $1^{\prime} 47^{\prime \prime}$ (range: $1^{\prime} 04^{\prime \prime}$ to $3^{\prime}$ ) [11].

In our findings, in 374 occasions of TBI, 82 players did not receive medical attendance. The mean time for the 292 players with TBIs who did receive assessment was $1^{\prime} 35^{\prime \prime}$ (range: $48^{\prime \prime}$ to $7^{\prime} 40^{\prime \prime}$ ). Considering only the 13 players who had a concussion and the 12 players who were replaced, the mean time for medical evaluation was $3^{\prime} 26^{\prime \prime}$ and $3^{\prime} 19$ ", respectively. If the two reported severe TBIs, which required an ambulance was on field, were excluded from the average, the mean time for medical evaluation goes down to $2^{\prime} 43$ " for players with concussion and 2' 54 " for replaced players after TBI. In comparison with the 2014 FIFA World Cup, there was an increased amount of time given for player assessment in the 2017 Brazilian Soccer Championship.

In November 2018, the Brazilian Soccer Confederation (CBF; Rio de Janeiro, Brazil) organized a Medical Symposium at Rio de Janeiro and discussed the protocols for concussion evaluation in Brazil. At this Symposium, the idea of a video review for concussion evaluation and an extra soccer player replacement were discussed [21]. 
Following this Symposium, the CBF started the discussion about medical training and protocols for concussion assessment, but no formal protocol for concussion assessment has been endorsed by CBF up to now [22].

The North American soccer league Major League Soccer implemented programs with video review and league spotters to provide medical staff detailed early detection of concussion. Major League Soccer data suggest that the video is effective in increasing knowledge on concussion. Major League Soccer, US Soccer and the National Women's Soccer League jointly hosted a conference on April 2017 in New York and discussed the temporary replacement of players with suspected concussion to allow time for adequate medical assessment [23].

Many sports, including basketball, rugby, American Football and ice hockey, have formal and feasible protocols for concussion. According to their rules, the athletes can be temporarily replaced to be evaluated on sideline and, after protocol tests, they can return to play or not.

Despite standardized protocols for concussion in soccer that have been endorsed by FIFA, the main concern that arises from recent studies, including this one, is that there is a gap between these formal protocols and the sideline medical assessment in practice. In addition, the recognition of injury and assessment always occur in a time-pressured environment, requiring rapid disposition and decision-making [5]. There is no doubt that the duration of this evaluation is very far from the ideal in order to preserve the player's integrity.

\section{Conclusion}

Soccer rules forbid temporary replacement of players during a match. This fact is potentially the biggest challenge in this kind of sport on how to deal with concussion assessment when compared with other team sports. It means that, in the soccer environment, the medical professionals are pressured to perform concussion protocols on the sideline as fast as possible because of a huge team disadvantage in the dynamic of the game without one player on the field. Our results among Elite Soccer Players in Brazil confirm that the length of medical evaluation on the sideline is not long enough for an adequate performance. Consequently, the concussion and other potential neurologic injuries may be misdiagnosed, with a life-threatening risk to the professional soccer player.

\section{Future perspective}

Considering the previous studies already mentioned, this report suggests an alternative to the challenging issue of soccer's replacement rules. This study supports the idea of creating a temporary replacement. Once a player is diagnosed with a potential concussion, the player with a concussion should be temporarily replaced, maintaining a team with all players on the field while the player is assessed. They can then return to play following a full medical evaluation, if it is medically advisable. This will provide time for an adequate sideline evaluation and preserve the integrity of soccer players, respecting the dynamic and the environment of the sport.

Executive summary

- Despite the limitations of video analysis, it may be a useful tool for recognizing possible concussion and it may be a useful adjunct to the sideline medical assessment.

- Traumatic brain injury is more often than expected and 'head-to-head' event is the majority of related head injury (48,6\%) among soccer players in 2017 Brazilian Championship.

- Among 13 players who had concussion, the unexpected ball hit was the main cause (four cases) and only four players were replaced after concussion, two of these were removed directly to hospital after impact seizure.

- The mean time for medical evaluation of the 13 players with concussion was 3'26". Despite standardized protocols for concussion in soccer endorsed by FIFA, the main concern that arises from this study is that there is a gap between these formal protocols and the sideline medical assessment in practice on Elite Soccer in Brazil.

- In the soccer environment, medical professionals are pressured to perform concussion protocols on the sideline as fast as possible because of a huge team disadvantage in the dynamic of the game without one player on the field. Our results confirm that the length of medical evaluation on the sideline is not enough for an adequate performance. Consequently, the concussion and other potential neurologic injuries may be misdiagnosed, with a life-threatening risk to the professional soccer player.

- This report supports the idea of creating a temporary replacement in soccer's replacement rules. This will allow time for an adequate sideline concussion evaluation and preserve the integrity of soccer players, respecting the dynamic and the environment of the sport. 
Financial \& competing interests disclosure

The authors have no relevant affiliations or financial involvement with any organization or entity with a financial interest in or financial conflict with the subject matter or materials discussed in the manuscript. This includes employment, consultancies, honoraria, stock ownership or options, expert testimony, grants or patents received or pending, or royalties.

No writing assistance was utilized in the production of this manuscript.

\section{Open access}

This work is licensed under the Creative Commons Attribution 4.0 License. To view a copy of this license, visit http://creativecommons.org/licenses/by/4.0/

\section{References}

Papers of special note have been highlighted as: $\bullet$ of interest

1. Harmon KG, Clugston JR, Dec K et al. American Medical Society for Sports Medicine position statement on concussion in sport. Br. J. Sports Med. 53(4), 213-225 (2019).

- The American Medical Society for Sports Medicine formed a writing group to study sports-related concussion and to address current evidence and knowledge gaps regarding sports-related concussion.

2. Bunc G, Ravnik J, Velnar T. May heading in football result in traumatic brain injury? A review of literature. Med. Arch. 71(5), 356-359 (2017).

3. Munia TTK, Haider A, Fazel-Rezai R. Evidence of brain functional deficits following sport-related mild traumatic brain injury. Annu. Int. Conf. IEEE Eng. Med. Biol. Soc. 2017, 3212-3215 (2017).

4. Putukian M. Clinical evaluation of the concussed athlete: a view from the sideline. J. Athl. Train. 52(3), 236-244 (2017).

5. McCrory P, Meeuwisse W, Johnston $\mathrm{K}$ et al. Consensus statement on concussion in sport: the 3rd International Conference on Concussion in Sport held in Zurich, November 2008. Br. J. Sports Med. 43(Suppl. 1), i76-i90 (2009).

6. McCrory P, Meeuwisse WH, Aubry M et al. Consensus statement on concussion in sport: the 4th International Conference on Concussion in Sport held in Zurich, November 2012. Br. J. Sports Med. 47(5), 250-258 (2013).

7. McCrory P, Meeuwisse WH, Dvořák J et al. Consensus statement on concussion in sport: the 5 th International Conference on Concussion in Sport held in Berlin, October 2016. Br. J. Sports Med. 51(11), 838-847 (2018).

8. Concussion in Sport Group. Sport Concussion Assessment Tool - 3rd Edition. Br. J. Sports Med. 47(5), 259 (2013).

9. Echemendia RJ, Meeuwisseet WH, McCrory P et al. Sport concussion assessment tool - 5th edition. Br. J. Sports Med. 51(11), 851-858 (2017).

- $\quad$ SCAT-5 is the protocol for concussion assessment endorsed by FIFA.

10. Abraham KJ, Casey J, Subotic A, Tarzi C, Zhu A, Cusimano MD. Medical assessment of potential concussion in elite football: video analysis of the 2016 UEFA European championship. BMJ Open 9(5), e024607 (2019).

- Shows that there is an urgent need to improve the assessment and management of players suspected of concussion in the realm of elite football.

11. Cusimano MD, Casey J, Jing R et al. Assessment of head collision events during the 2014 FIFA world cup tournament. JAMA 317(24), 2548-2559 (2017).

- In the 2014 World Cup, concussion assessment protocols were not followed in $63 \%$ of events when players involved in head collisions were not assessed by sideline health care personnel.

12. Makdissi M, Davis G. The reliability and validity of video analysis for the assessment of the clinical signs of concussion in Australian football. J. Sci. Med. Sport. 19(10), 859-863 (2016).

- Video analysis may be a useful adjunct to the sideline assessment of possible concussion but should not replace the need for a thorough multimodal assessment.

13. Bjørneboe J, Bahr R, Andersen TE. Video analysis of situations with a high-risk for injury in Norwegian male professional football; a comparison between 2000 and 2010. Br. J. Sports Med. 48(9), 774-778 (2014).

14. Gardner A, Iverson G, Wojtowicz $\mathrm{M}$ et al. A systematic video analysis of concussion in the National Rugby League. Neurology 84(Suppl. 14), P7.165 (2015).

- First study to use video surveillance to examine concussion in National Rugby League in Australia.

15. Fédération Internationale de Football Association. WORLD CUP USA '94: ROUND OF 16: Leonardo Says Elbow Wasn't Intentional: Brazil: he apologizes to Ramos but might have to sit out rest of tournament. Los Angeles Times (1994). www.latimes.com/archives/la-xpm-1994-07-06-sp-12328-story.html

16. Premier League. Petr Cech sustained a fractured skull 10years ago in collision with Stephen Hunt. a look back at horror incident which changed the goalkeeper's life. The Daily Mail (2016). www.dailymail.co.uk/sport/football/article-3838113/Petr-Cech-sustained-fractur ed-skull-10-years-ago-collision-Stephen-Hunt-look-incident-changed-goalkeeper-s-life.html 
17. La Liga. Fernando Torres hospitalized after suffering serious head injury. Vice (2017). www.vice.com/en/article/ezeyep/fernando-torres-hospitalized-after-suffering-serious-head-injury

18. Paranaense Federation of Soccer. Morte do primeiro capitão do Paraná completa 25 anos e deixa rastro de dor, revolta e dívida. Gazeta Do Povo (2015). www.gazetadopovo.com.br/esportes/futebol/parana-clube/morte-do-primeiro-capitao-do-parana-completa-25-anos-e -deixa-rastro-de-dor-revolta-e-divida-dhoh2incjqd9xyil8mzdqlnll/

19. Elbin RJ, Zuckerman SL, Sills AK, Crandall JR, Lessley DJ, Solomon GA. Sensitivity and specificity of on-field visible signs of concussion in the National Football League. Neurosurgery 87(3), 530-537 (2020).

20. Caccese JB, Kaminski TW. Minimizing head acceleration in soccer: a review of the literature. Sports Med. 46(11), 1591-1604 (2016).

21. Mooney J, Self M, ReFaey K et al. Concussion in soccer: a comprehensive review of the literature. Concussion 5(3), CNC 76 (2020).

22. Fourth Medical Symposium of Brazilian Football Confederation. Jorge Pagura debates brain concussion protocol at the Medical Symposium. Brazilian Football Confederation (2018).

www.cbf.com.br/a-cbf/informes/comissao-medicos/jorge-pagura-debate-protocolo-de-concussao-cerebral-no-simposio-medico

23. Putukian M, Echemendia RJ, Chiampas G et al. Head injury in soccer: from science to the field; summary of the head injury summit held in April 2017 in New York City, New York. Br. J. Sports Med. 53(21), 1332 (2019). 